\title{
ANALYSIS OF SMALL-SIGNAL PARAMETERS OF 2-D MODFET WITH POLARIZATION EFFECTS FOR MICROWAVE APPLICATIONS
}

\author{
Ramnish Kumar ${ }^{1}$, Sandeep K Arya ${ }^{1}$ and Anil Ahlawat ${ }^{2}$ \\ ${ }^{1}$ Department of ECE, GJUST, Hisar \\ ${ }^{2}$ Department of CSE, KIET, Ghajiabad \\ contactram1@rediffmail.com
}

\begin{abstract}
An improved analytical two dimensional (2-D) model for AlGaN/GaN modulation doped field effect transistor (MODFET) has been developed. The model is based on the solution of 2-D Poisson's equation. The model includes the spontaneous and piezoelectric polarization effects. The effects of field dependent mobility, velocity saturation and parasitic resistances are included in the current voltage characteristics of the developed two dimensional electron gas (2-DEG) model. The small-signal microwave parameters have been evaluated to determine the output characteristics, device transconductance and cut-off frequency for $50 \mathrm{~nm}$ gate length. The peak transconductance of $165 \mathrm{mS} / \mathrm{mm}$ and a cut-off frequency of $120 \mathrm{GHz}$ have been obtained. The results so obtained are in close agreement with experimental data, thereby proving the validity of the model.
\end{abstract}

\section{KEYWORDS}

AlGaN/GaN MODFETs, cut-off frequency, drain - conductance, polarization, trans - conductance.

\section{INTRODUCTION}

In recent years, MODFETs (Modulation Doped Field Effect Transistors) or HEMTs (High Electron Mobility Transistors) have been developed because of their very high switching speed, low power consumption and relatively simple fabrication technology. The HEMT fabricated in $\mathrm{AlGaN} / \mathrm{GaN}$ materials is most suitable for high power, low noise, high speed, good stability and high temperature microwave devices. The use of HEMTs is increasing in many microwave circuits and systems because of their high frequency and high speed response. The pseudomorphic high electron mobility transistors have shown excellent microwave and noise performance and are very attractive for millimetre wave and optoelectronic applications [1-3]. Recently, pHEMTs have shown superior performance at microwave and millimetre frequency range. pHEMTs have also demonstrated excellent performance, both as microwave and digital devices [4-6]. AlGaN/GaN HEMTs have emerged as a strong option for high power application owing to their large band gap energy and high saturation velocity [7-9]. The presence of strong polarization (spontaneous \& piezoelectric) fields leads to the enhanced performance of these devices. The polarization charges, the conduction-band discontinuity and mole fraction are the important parameters that affect the sheet carrier density at the interface. An increase in aluminium composition in the mole fraction of $\mathrm{AlGaN} / \mathrm{GaN}$ pHEMTs increases the density of the two dimensional electron gas and electron lie more closely to the interface. The additional characteristic features of the $\mathrm{AlGaN} / \mathrm{GaN}$ material that lead to excellent performance of $\mathrm{GaN}$ - 
International Journal of VLSI design \& Communication Systems (VLSICS) Vol.4, No.2, April 2013

based HEMTs are large breakdown field and high thermal stability [10]. Along with advances in HEMT fabrication, large number of analytical and numerical models has been developed [11-22]. These models are helpful as they provide good insight into the physical operation of the device. But, they normally require some simplifying assumptions to obtain the sheet charge density of 2DEG. The present model is developed by solving the two dimensional poisson's equation and the parasitic resistance. This model is then used to derive the small-signal parameters namely transconductance, drain conductance, transit time and cut-off frequency of AlGaN/GaN pHEMTs, including the effects of spontaneous and polarization fields. The 2-D analysis of the device has been carried out in the saturation region and modified expression of device transconductance and output conductance has been given. The results of the proposed model have been verified with the published experimental/ simulated data.

\section{THEORETICAL CONSIDERATION}

The basic structure of AlGaN/GaN pHEMT considered in the present analysis is [11] as shown in figure-1.

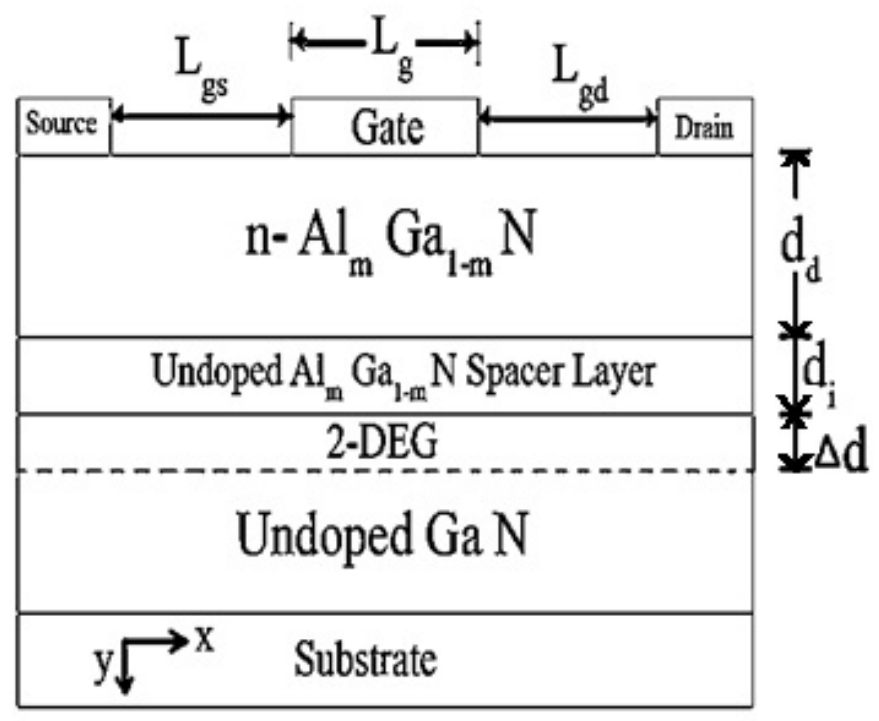

Figure 1.Schematic diagram of $\mathrm{AlGaN} / \mathrm{GaN}$ pHEMT

The 2-DEG sheet charge density formed at the $\mathrm{Al}_{\mathrm{m}} \mathrm{Ga}_{1-\mathrm{m}} \mathrm{N} / \mathrm{GaN}$ heterointerface is obtained By solving Poisson's equation [12-13] as

$n_{s}(m, x)=\frac{\varepsilon(m)}{q \cdot\left(d_{d}+d_{i}+\Delta_{d}\right)}\left(V_{g s}-V_{c}(x)-V_{t h}(m)\right)$

Where

$\mathrm{d}_{\mathrm{d}=}$ doped $\mathrm{AlGaN} / \mathrm{GaN}$ Layer thickness

$\mathrm{d}_{\mathrm{i}}=$ spacer (undoped $\mathrm{AlGaN} / \mathrm{GaN}$ ) layer thickness

$\Delta_{\mathrm{d}}=$ effective thickness of 2-DEG

$\mathrm{D}=\mathrm{d}_{\mathrm{d}}+\mathrm{d}_{\mathrm{i}}+\Delta_{\mathrm{d}}=$ separation between the gate and the channel

$\mathrm{q}=$ electronic charge

$\mathrm{m}=\mathrm{Al}$ mole fraction

$\mathcal{E}(\mathrm{m})=\mathrm{AlGaN} / \mathrm{GaN}$ dielectric constant 
$\mathrm{V}_{\mathrm{gs}}=$ applied gate source voltage

$\mathrm{V}_{\mathrm{c}}(\mathrm{x})=$ channel potential at $\mathrm{x}$ due to the drain voltage.

$\mathrm{V}_{\mathrm{th}}=$ threshold voltage.

Threshold voltage is defined as the applied gate voltage for which the channel is completely depleted of free carriers and is considered as the minimum potential in the channel. The threshold voltage $\mathrm{V}_{\mathrm{th}}(\mathrm{m})$ of $\mathrm{AlGaN} / \mathrm{GaN}$ pHEMT is strongly dependent on polarization charge density. It is given [16] as:

$V_{t h(m)}=\Phi m(m)-\Delta E_{c}(m)-\frac{q N_{d} d_{d}^{2}}{2 \varepsilon(m)}-\frac{D \cdot \sigma(m)}{\varepsilon(m)}+\frac{E_{f(m)}}{q}$

Where

$\Phi_{\mathrm{m}}(\mathrm{m})=$ schottky barrier carrier

$\Delta \mathrm{E}_{\mathrm{c}}(\mathrm{m})=$ conduction-band discontinuity of $\mathrm{AlGaN} / \mathrm{GaN}$ interface

$\mathrm{N}_{\mathrm{d}}=$ doping concentration of the $\mathrm{AlGaN}$ barrier

$\sigma(\mathrm{m})=$ net polarization induced sheet charge density at the AlGaN/GaN interface

$\mathrm{E}_{\mathrm{f}}(\mathrm{m})=$ Fermi potential

The total polarization induced charge sheet density is given [10] as:

$\sigma(m)=P_{S P}\left(A l_{m} G a_{1-m} N\right)-P_{S P}(G a N)+P_{P Z}\left(A l_{m} G a_{1-m} N\right)-P_{P Z}(G a N)$

Where

$\mathrm{P}_{\mathrm{SP}}=$ Spontaneous polarization of $\mathrm{AlGaN}$ and $\mathrm{GaN}$ layers resp.

$\mathrm{P}_{\mathrm{PZ}}=$ Piezoelectric polarization of $\mathrm{AlGaN}$ and $\mathrm{GaN}$ resp.

In the above expression, it has been assumed that the GaN layer is fully relaxed. This is reasonable assumption since the thickness of $\mathrm{GaN}$ layer is much larger than that of strained $\mathrm{AlGaN}$ layer. Thus $\mathrm{Ppz}=0$.

The total amount of polarization induced sheet charge density for $\mathrm{AlGaN} / \mathrm{GaN}$ heterostructure field effect transistor is obtained as:

$|\sigma(m)|=\left|P_{S P}\left(A l_{m} G a_{1-m} N\right)-P_{S P}(G a N)+P_{P Z}\left(A l_{m} G a_{1-m} N\right)\right|$

Where

$P_{P Z}\left(A l_{m} G a_{1-m} N\right)=2\left(\frac{a(o)-a(m)}{a(m)}\right)\left(e_{31}(m)-e_{33}(m) \frac{c_{13}(m)}{c_{33(m)}}\right)$

$\mathrm{P}_{\mathrm{sp}}\left(A l_{m} G a_{1-m} N\right)=-0.052 \mathrm{~m}-0.029$

$\operatorname{Psp}(\mathrm{GaN})=-0.029$

a (m) is lattice constant, $e_{31}(m)$ and $e_{33}(m)$ are piezoelectric constants, $c_{13}(m)$ and $c_{33}(m)$ are elastic constants respectively. 
International Journal of VLSI design \& Communication Systems (VLSICS) Vol.4, No.2, April 2013

\subsection{CURRENT -VOLTAGE CHARACTERISTICS}

The drain source current in the channel is obtained from the current density equation and is given [15] as:

$I_{d s}(m, x)=z q \mu(x, m)\left(n_{s}(m, x) \frac{d v_{C}(x)}{d x}+\frac{k_{B} T}{q} \frac{d n_{S}(m, x)}{d x}\right)$

Where $\mathrm{z}$ is the gate width, $\mathrm{T}$ is temperature, $\mathrm{K}_{\mathrm{B}}$ is Boltzman constant.

$\mu(\mathrm{x})=$ field dependent electron mobility and is given [11] as:

$\mu(x, m)=\frac{\mu_{0}(m)}{1+\left(\frac{\mu_{0}(m) E_{c-} V_{s a t}}{E_{c} V_{s a t}}\right) \frac{d v_{c x}}{d x}}$

Using equations (1) and (7) in (6) and on integrating using boundary conditions

$\left.v_{c}(x)\right|_{x=0}=I_{d s}(m, x) R s$

$\left.v_{c}(x)\right|_{x=L}=v_{d s}-I_{d s}(m, x)(R s+R d)$

Where $\mathrm{Rs}$ and $\mathrm{Rd}$ are the parasitic source and drain resistances respectively.

The $\mathrm{I}_{\mathrm{ds}}-\mathrm{V}_{\mathrm{ds}}$ equation for the linear region is obtained as

$I_{d s}=\frac{-\alpha \pm \sqrt{\alpha^{2}-4 \beta \gamma}}{2 \beta}$

Where

$$
\begin{aligned}
& \alpha=-\left[\mathrm{L}+\mathrm{E}_{1} \mathrm{~V}_{\mathrm{ds}}+\mathrm{E}_{2}\left(\mathrm{~V}_{\mathrm{gs}-} \mathrm{V}_{\mathrm{th}}-\frac{\mathrm{k}_{\mathrm{B}} \mathrm{T}}{\mathrm{q}}\right)(2 \mathrm{Rs}+\mathrm{Rd})-\mathrm{E}_{2} \mathrm{~V}_{\mathrm{ds}}(\mathrm{Rs}+\mathrm{Rd})\right] \\
& \beta=E_{1}(2 R s+R d)-\frac{E_{2} R d}{2}(R d+2 R s) \\
& \gamma=E_{2}\left(V_{g s-} V_{t h}-\frac{k_{B} T}{q}\right) V_{d s}-\frac{E_{2}}{2} V_{d s}^{2} \\
& \frac{\mu_{o} E_{c}-V_{s a t}}{E_{c} V_{s a t}}=E_{1} \\
& \frac{Z \mu_{o} \varepsilon(m)}{D}=E_{2} \\
& \frac{d v_{c}(x)}{d x}=V_{c}^{\prime}(x)
\end{aligned}
$$

At the onset of saturation, the carriers get velocity saturated, and the electric field attains the critical value $\left(E_{c}\right)$. The current in the saturation region is obtained as

$$
I_{d s a t}=\frac{Z \mu_{o} E_{c} \varepsilon(m)}{d}\left[V_{g s-} V_{t h-} V_{d s a t}(m)-\frac{k_{B} T}{q}\right]
$$


The saturation current can also be obtained from equation (10) by replacing $\mathrm{V}_{\mathrm{ds}}$ by $\mathrm{V}_{\mathrm{dsat}}$. The drain saturation voltage $V_{d s a t}$ is obtained by equating the two expressions for $I_{d s a t}$ due to the current continuity between the linear and saturation region as by equations (10) and (17):

$\mathrm{V}_{\mathrm{dsat}}=\frac{-\Phi_{1} \pm \sqrt{\Phi_{1}^{2}-4 \Phi_{2} \Phi_{3}}}{2 \Phi_{2}}$

Where

$$
\begin{aligned}
& \Phi_{1}=\frac{\mu_{o} Z \varepsilon(m) E_{c}}{d}\left[\frac{-2 Q \beta \mu_{o} Z \varepsilon(m) E_{c}}{d}-E_{1} Q+E_{2} Q(R s+R d)+L+E_{2} Q(2 R s+R d)\right]+ \\
& E_{2} Q \\
& \Phi_{2}=\frac{\mu_{o} Z \varepsilon(m) E_{c}}{d}\left[\frac{\beta \mu_{o} Z \varepsilon(m) E_{c}}{d}+E_{1}-E_{2}(R s+R d)\right]-\frac{E_{2}}{2} \\
& \Phi_{3}=\frac{Q \mu_{o} Z \varepsilon(m) E_{c}}{d}\left[\frac{\beta \mu_{o} Z \varepsilon(m) E_{2} Q}{d}-L-\frac{E_{2} Q(2 R s+R d)}{d}\right] \\
& Q=V_{g s-} V_{t h}-\frac{K_{B} T}{q}
\end{aligned}
$$

\subsection{SMALL SIGNAL PARAMETERS}

The small signal parameters (drain conductance, transconductance, cut-off frequency and transit time) govern the current driving capability and are extremely important for estimating the microwave performance of the device[11,12]. The small signal parameters have been modelled in terms of basic device parameters and terminal voltages to give an insight into device performance and serve as a basis for device design and optimization.

\section{(a) Drain/Output conductance}

It is an important microwave parameter that determines the maximum voltage gain attainable from a device. The drain conductance of the $\mathrm{AlGaN} / \mathrm{GaN}$ pHEMT is evaluated as

$$
g_{d}(m)=\frac{\partial I_{d s}(m)}{\partial V_{d s}} \text { at constant } V_{g s}
$$

$g_{d}(m)=\frac{1}{2 \beta}\left[-E_{1}+E_{2}\left(R_{d}+R_{S}\right)+\frac{1}{2 \sqrt{\alpha^{2}-4 \beta \gamma}}\left\{2 \alpha\left(-E_{1}+E_{2}\left(R_{d}+R_{S}\right)\right)-4 \beta E_{2}(Q-\right.\right.$ $\left.\left.\left.V_{d s}\right)\right\}\right]$

\section{(b) Transconductance}

It is the most important parameter for optimization of FET high frequency behaviour. The major part of the gain mechanism is embodied in the active channel transconductance, which is evaluated as

$g_{m}(m)=\frac{\partial I_{d s}(m)}{\partial V_{g s}}$ at constant $V_{d s}$ 
International Journal of VLSI design \& Communication Systems (VLSICS) Vol.4, No.2, April 2013

$g_{m}(m)=\frac{1}{2 \beta}\left[-E_{2}(2 R s+R d)+\frac{1}{2 \sqrt{\alpha^{2}-4 \beta \gamma}}\left\{2 \alpha\left(-E_{2}(2 R s+R d)\right)-4 \beta E_{2} V_{d s}\right\}\right]$

\section{(c) Cut-off frequency}

The primary figure of merit for high frequency performance of a device is the current gain cutoff frequency. The cut-off frequency of the AlGaN/GaN MODFET is calculated as

$f_{t}(m)=\frac{g_{m}(m) D}{2 \pi Z \varepsilon(m)}$

By substituting the value of trans-conductance from equation (26), the cut-off frequency can be evaluated.

\section{(d) Transit time}

The transit time effect is the result of a finite time being required for carriers to traverse from source to drain. Smaller transit times are desirable to attain a high frequency response from a device. The transit time for the AlGaN/GaN pHEMT is evaluated as

$T_{t}(m)=\frac{1}{2 \pi f_{t}(m)}$

By putting equation (27) in equation (28), transit time can be obtained.

\section{RESULTS AND DISCUSSION}



Figure 2. Current-voltage characteristics of $\mathrm{AlGaN} / \mathrm{GaN}$ HEMT for 50nm gate Length

Figure 2 shows the current-voltage characteristics of AlGaN/GaN HEMT for various values of gate source voltages. It can be seen that current increases with the increase in drain source 
voltage. The device have a maximum drain current density of $501.5 \mathrm{~mA} / \mathrm{mm}$ at a gate bias of $1 \mathrm{~V}$ and a drain bias of $6 \mathrm{~V}$.High currents are attributed to very high sheet charge density, resulting from large conduction band discontinuity and strong polarization effects. The calculations for drain currents have been done for $\mathrm{Al}$ mole fraction $(\mathrm{m})$ equal to 0.15 . This shows that AlGaN/GaN devices can be effectively used for high power applications.it is seen that it resembles with the drain characteristics of MOSFET and the drain current gets saturated at an applied voltage of $4 \mathrm{~V}$. The results are in good agreement with the previously published experimental data.

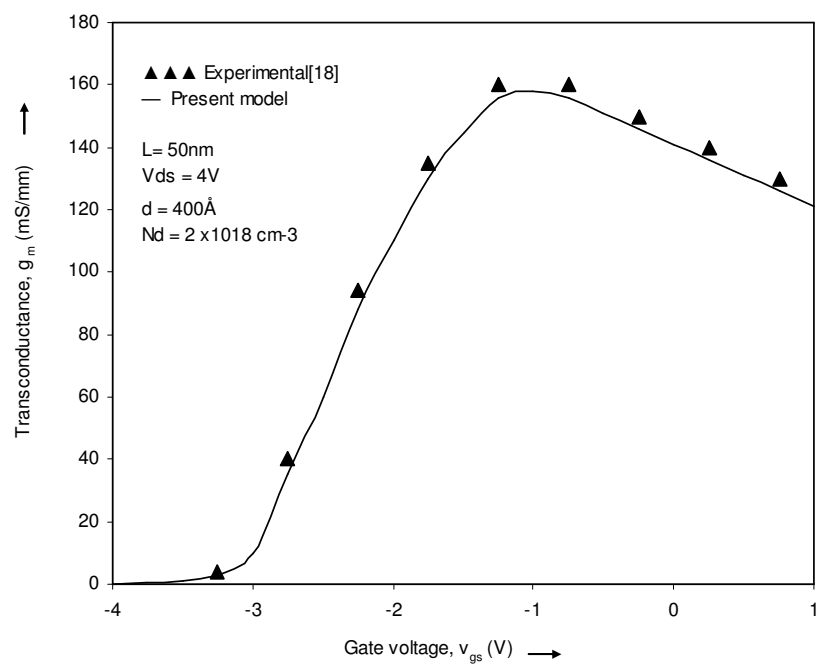

Figure 3. Variation of transconductance with gate source voltage

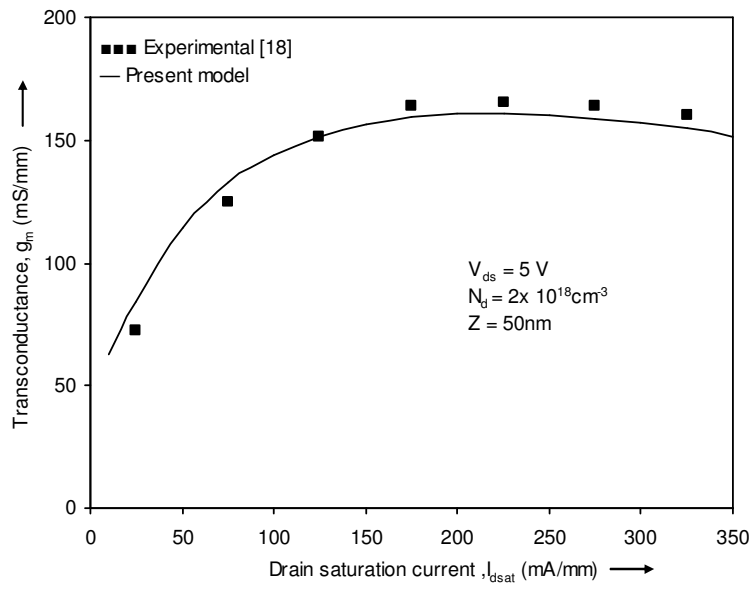

Figure 4. Variation of transconductance with drain current

Figure 3 shows the peak value of transconductance which occurs near the gate bias at which the 2-DEG charge density reaches the equilibrium value is $159 \mathrm{mS} / \mathrm{mm}$ at a gate bias of $-1 \mathrm{~V}$. The high transconductance may be attribute to the improved charge control and better transport properties in the GaN based HEMT. The decrease in transconductance at higher values of $\mathrm{V}_{\mathrm{gs}}$ occurs, because with the 2-DEG density approaches the equilibrium value, the current density no longer increases proportionally with the gate voltage. The results of the model are in close agreement 
with the experimental data. The proposed model is valid over a large range of gate lengths and widths and is thus highly suitable for device structure and performance optimization.

Figure 4 shows the variation of transconductance with the drain saturation current. The transconductance increases for smaller values of current and then saturate to peak value of $165 \mathrm{mS} / \mathrm{mm}$ and a drain current of $220 \mathrm{~mA} / \mathrm{mm}$. The results are in close proximity with the experimental data which confirms the validity of the proposed model



Figure 5. Variation of cut-off frequency with gate length

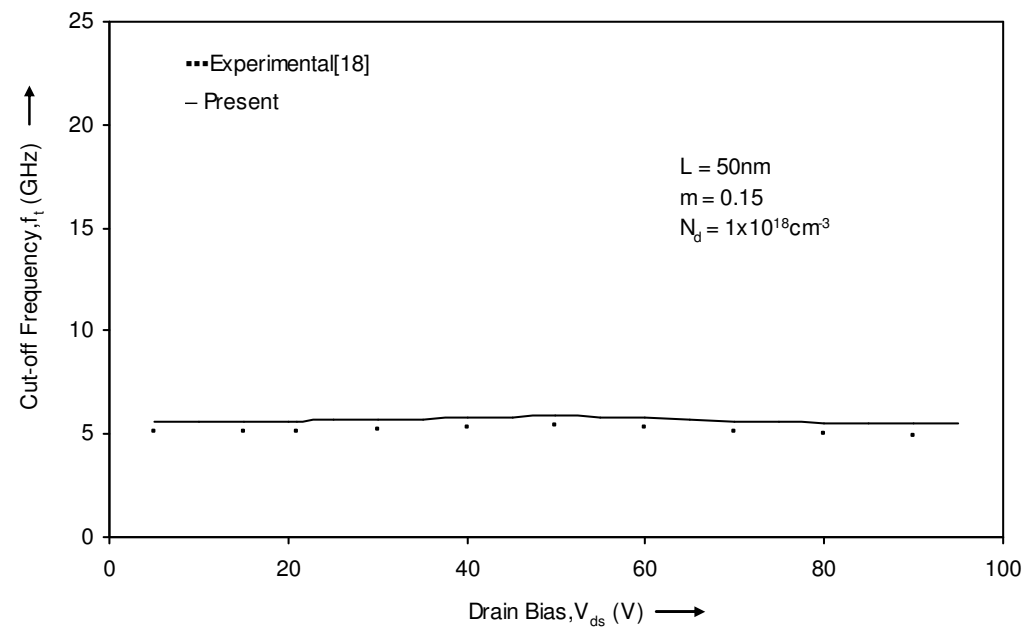

Figure 6. Current gain cut-off frequency with drain voltage

Figure 5 shows the variation of cut-off frequency with gate length. Ft falls sharply with an increase in the gate length. As the channel length is increased, the electron transit time through the channel also increases, thus causing a reduction of the frequency. A high cut-off frequency of about $122 \mathrm{GHz}$ is obtained at a gate length of $50 \mathrm{~nm}$. As compared with experimental data, cut-off frequency increases with the decrease in gate length. 
Figure 6 shows the variation of cutoff frequency with drain bias. The cutoff frequency of about $5.2 \mathrm{GHz}$ at a drain bias of $40 \mathrm{~V}$ is obtained for the present proposed model, which indicates its higher microwave power ability. As the bias voltage increases, the cutoff frequency exhibits a slight increase. The results confirm the validity of the proposed model.

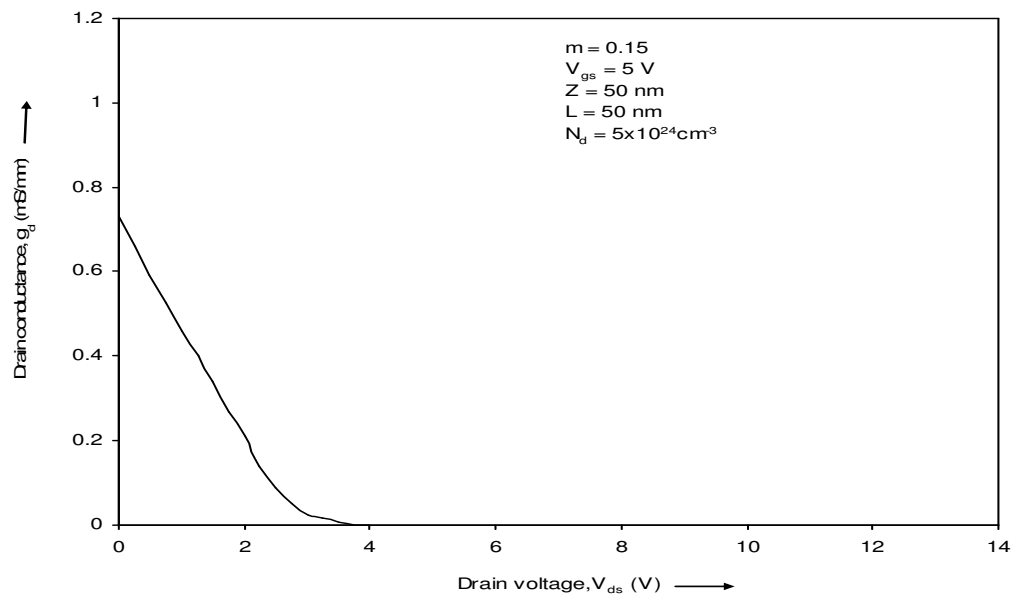

Figure 7. variation of drain conductance with drain voltage

Figure 7 shows the variation of output conductance with drain voltage. The drain conductance decreases with an increase in the drain bias until it becomes zero in saturation region. This happens because with an increase in the drain bias voltage, the carrier velocity rises gradually and then saturates. The results are in good agreement with the previously published results.

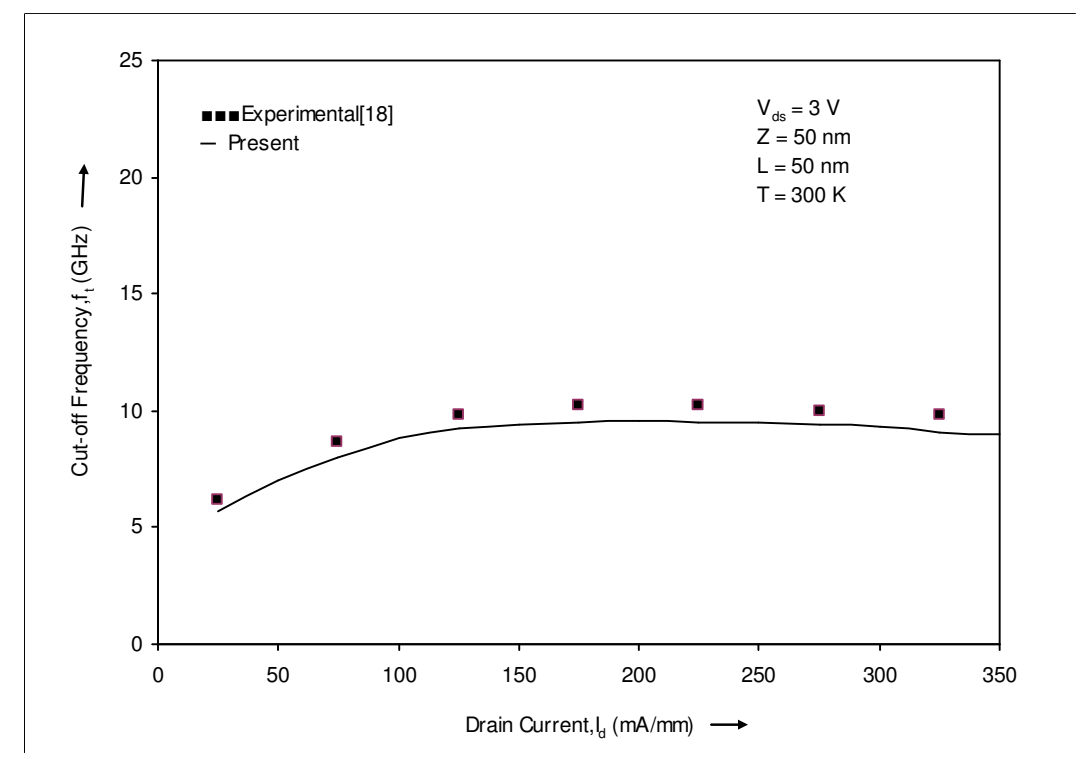

Figure 8.Variation of cut-off frequency with drain current

Figure 8 shows the variation of cut-off frequency with drain current density. A cut-off frequency of $9.5 \mathrm{GHz}$ is obtained at a drain current of $185 \mathrm{~mA} / \mathrm{mm}$. when drain current is low, transconductance is low and hence cut-off frequency is also low. And when transconductance is high means drain current is high means cut-off frequency is also high. The results are in good agreement with the experimental results. 
International Journal of VLSI design \& Communication Systems (VLSICS) Vol.4, No.2, April 2013

\section{CONCLUSION}

The proposed model is developed for the 2 DEG sheet charge density which is the most important parameter in characterizing and evaluating the performance of AlGaN/GaN pHEMTs. The model is developed for the I-V characteristics and small signal parameters of an AlGaN/GaN MODFET taking into effect of strong polarization effects. The model also shows the potential of $\mathrm{AlGaN} / \mathrm{GaN}$ pHEMT as a future candidate for high power, high speed applications. The model can further be extended to obtain the device capacitances and noise characteristics.

\section{REFERENCES}

[1] Z. Hemaizia et al. (2010), "Small-signal modelling of phemts \& analysis of their microwave performance", Universite Mohamed Khider, Biskra, Vol. 10, pp 59-64.

[2] M. N. Yoder, (1997) "Gallium nitride: Past, present and future", Int. Electron Devices Meeting Technical Dig., pp3-12.

[3] M. S. Shur, (1998) "GaN based transistors for high power applications", Solid State Electron., Vol. 42, No.12, pp2131-2138,

[4] A.Asgari et al., (2005) "Theoretical model of transport characteristics of AlGaN/GaN high electron mobility transistors" WILEY, phys. Stst. Sol. (c), no.3, 1047-1055(2005).

[5] P.M. smith, P.C. Chao, J.M. Ballingall, and A.W. Swanson, (1990) "Micro-wave and mm-wave power application using PHEMTs", Microwave J., pp71- 86.

[6] C.S. Wu, F. Ren, S.J. Pearton, M. Hu, C.K. Pao, and R.F. Wang, (1995) "High efficiency microwave power AlGaAs/InGaAs PHEMT's fabricated by dry etch single gate recess”, IEEE Trans Ed 42, pp1419-1424.

[7] Y. Zhang, I. P. Smorchkova, C. R. Elsass, S. Keller, J. P. Ibbetson, S.Denbaars, U. K. Mishra, and J. Singh, (2000) "Charge control and mobility inAlGaN/GaN transistors: Experimental and theoretical studies," J. Appl.Phys., Vol. 87, No. 11, pp7981-7987.

[8] R. Anholt, Electrical and thermal characterization of MESFETs,HEMTs and HBTs, Artech House, Boston, 1995.

[9] Jonathan C. Sippel et al. (2007) "A physics based model of DC and microwave characteristics of GaN/AlGaN HEMTs”, International journal of RF and Microwave Computer aided Engineering, , WILEY INTERSCIENCE, DOI 10.1002/mmce.

[10] O. Ambacher, J. Smart, J. R. Shealy, N. G. Weimann, K. Chu, M.Murphy, W. J. Schaff, L. F. Eastman, R. Dimitrov, L. Wittmer, M.Stutzmann, W. Rieger, and J. Hilsenbeck, (1999) “Twodimensional electron gases induced by spontaneous and piezoelectric polarization charges in $\mathrm{N}$ - and Ga-face AlGaN/GaN heterostructures”, J. Appl. Phys., Vol. 85, No. 6, pp 3222-3233.

[11] Parvesh Gangwani et al., (2007) "Polarization dependent analysis of AlGaN/GaN HEMT for high power applications", Elsevier, ScienceDirect, Solid-State Electronics 51, pp130-135.

[12] Rashmi, A. Kranti, S. Haldar, and R. S. Gupta, (2002) "An accurate charge control model for spontaneous and piezoelectric polarization dependent two-dimensional electron gas (2-DEG) sheet charge density of lattice mismatched AlGaN/GaN HEMTs," Solid State Electron., Vol. 46, No. 5, pp $621-630$.

[13] Rajesh K. Tyagi et al., (2007) "An analytical two-dimensional model for AlGaN/GaN HEMT with polarization effects for high power applications”, Elsevier, Microelectronics Journal, 38, pp877-883.

[14] C.S. Chang and D.Y.S. Day, (1989) "Analytic theory for current voltage characteristics and field distribution of GaAs MESFETs", IEEE Trans Electron Devices 36, pp269 -280.

[15] H. Rohdin and P. Robin, (1986) “A MODFET dc model with improved pin-choff and saturation characteristics, IEEE Trans Electron Devices ED, 33, pp664 - 672.

[16] Anil Ahlawat et al., (2007) "Microwave analysis of $70 \mathrm{~nm}$ InGaAs pHEMT on InPsobstrate for nanoscale digital IC application”, Microwave and Optical technology letters, Vol. 49, No. 10, pp 2462-2470.

[17] Rajesh K. Tyagi et al., (2009) "Noise analysis of sub quarter micrometer AlGaN/GaN microwave power HEMT", JSTS, Vol.9, No.3, pp125-135. 
International Journal of VLSI design \& Communication Systems (VLSICS) Vol.4, No.2, April 2013

[18] Y.F. Wu, S. Keller, P. Kozodoy, B.P. Keller, P. Parikh, D. Kapolnek,S.P. Denbaars, U.K. Mishra, (1997) "Bias dependent microwave performanceof AlGaN/GaN MODFET's up to $100 \mathrm{~V}$ ", IEEE Electron. Dev. Lett.18, pp290-292.

[19] Ruediger Quay et al., (2001) "Nonlinear electronic transport and device performance of HEMTs", IEEE transactions on electron devices, Vol.48, No.2, pp210-217.

[20] C.S. Chang, H.R. Fetterman, (1987) “An analytical model for HEMTs using new velocity field dependence”, IEEE Trans. ED, Vol. ED- 34, 1456-1462.

[21] A. Agarwal, A. Goswami, S. Sen, and R.S. Gupta, (1999) "Capacitance-voltage characteristics and cutoff frequency of pseudomorphic (Al-GaAs/InGaAs) modulation-doped field-effect transistor for microwaveand high-speed circuit applications", Microwave Opt Technol Lett 23, pp312-318.

[22] V.Kumar, W Lu, R.Schwindt, A. Kuliev, G. Simin, J.Yang, M.A.Khan, and H.Adesida, (2002) "AlGaN/GaN HEMT in SiC with fT of over 120GHz", IEEE Elect. Dev. Lett 23, pp455-457. 